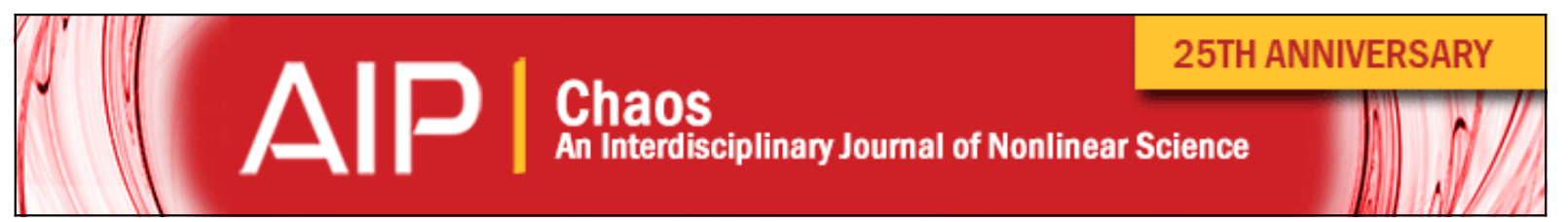

\title{
Exact period-four solutions of a family of $n$-dimensional quadratic maps via harmonic balance and Gröbner bases
}

María Belén D'Amico and Guillermo L. Calandrini

Citation: Chaos 25, 113113 (2015); doi: 10.1063/1.4935955

View online: http://dx.doi.org/10.1063/1.4935955

View Table of Contents: http://scitation.aip.org/content/aip/journal/chaos/25/11?ver=pdfcov

Published by the AIP Publishing

\section{Articles you may be interested in}

Caputo standard a-family of maps: Fractional difference vs. fractional

Chaos 24, 023137 (2014); 10.1063/1.4885536

On the determination of the limit cycles using the harmonic balance method

J. Math. Phys. 54, 103510 (2013); 10.1063/1.4826105

Four dimensional chaos and intermittency in a mesoscopic model of the electroencephalogram

Chaos 23, 023111 (2013); 10.1063/1.4804176

Analysis of stable periodic orbits in the one dimensional linear piecewise-smooth discontinuous map

Chaos 22, 033126 (2012); 10.1063/1.4740061

Yet another 3D quadratic autonomous system generating three-wing and four-wing chaotic attractors Chaos 19, 013107 (2009); 10.1063/1.3070648

\section{Cross-pollinate.}




\title{
Exact period-four solutions of a family of $n$-dimensional quadratic maps via harmonic balance and Gröbner bases
}

\author{
María Belén D’Amico ${ }^{1, a)}$ and Guillermo L. Calandrini ${ }^{1,2, b)}$ \\ ${ }^{1}$ Instituto de Investigaciones en Ingeniería Eléctrica "Alfredo Desages" (UNS-CONICET), \\ Departamento de Ingeniería Eléctrica y de Computadoras, Universidad Nacional del Sur, Avda. Alem 1253, \\ B8000CPB Bahía Blanca, Argentina \\ ${ }^{2}$ Departamento de Matemática, Universidad Nacional del Sur, Avda. Alem 1253, B8000CPB Bahía Blanca, \\ Argentina
}

(Received 9 January 2015; accepted 5 November 2015; published online 23 November 2015)

\begin{abstract}
Analytical solutions of the period-four orbits exhibited by a classical family of $n$-dimensional quadratic maps are presented. Exact expressions are obtained by applying harmonic balance and Gröbner bases to a single-input single-output representation of the system. A detailed study of a generalized scalar quadratic map and a well-known delayed logistic model is included for illustration. In the former example, conditions for the existence of bistability phenomenon are also introduced. (c) 2015 AIP Publishing LLC. [http://dx.doi.org/10.1063/1.4935955]
\end{abstract}

Period-four orbits exhibited by a family of $\boldsymbol{n}$-dimensional quadratic maps are analyzed via a frequency-domain (FD) viewpoint. The methodology relies on the inputoutput representation of the system, the Fourier series decomposition of the orbit, and the application of the harmonic balance. Contrary to the state-space representation, the proposed input-output description reduces to the interaction of two scalar functions, facilitating the developments. Analytical and exact expressions of the periodic points are found by applying Gröbner bases to a set of quadratic polynomial equations. The appearance of these oscillations in a generalized scalar quadratic map and a well-known delayed logistic model is studied in detail. In the last example, results related to the appearance of the bistability phenomenon are also obtained. In particular, it is shown that a period-four attractor can coexist with a stable fixed point/period-two orbit only if delayed samples are congruent with one (modulus four). In addition, it is illustrated how two stable period-four orbits can exist simultaneously for even delays congruent with two (modulus four).

\section{INTRODUCTION}

There exists a great variety of discrete maps coming from different branches of science that manifest the appearance of periodic orbits. The origin of these maps lies in the representation of temporal evolutions that are inherently discrete as well as the analysis of oscillations in continuous dynamical systems via the Poincaré section. Among the extensive list of examples, it can be mentioned the population models in Biology, ${ }^{1-3}$ the cardiac activity models in Medicine, ${ }^{4-6}$ the structure markets in Economics, ${ }^{7,8}$ the impact systems in Mechanics, ${ }^{9,10}$ the modulated lasers in Physics, ${ }^{11-13}$ the power converters in Electronics, ${ }^{14,15}$ etc. In many of these applications, quadratic maps have played an

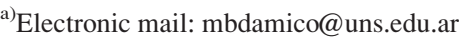

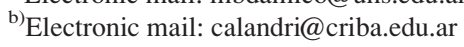

important role in the description of the detected dynamical scenarios.

Discrete maps can undergo periodic orbits due to existence of period-doubling bifurcations, Neimark-Sacker bifurcations, and even more complicated nonlinear phenomena such as period-doubling cascades, ${ }^{16,17}$ weak and strong resonances, ${ }^{18}$ bubbles, ${ }^{19}$ bistability, ${ }^{11-13}$ and periodic windows after the onset of chaos. ${ }^{20,21}$ Most of these scenarios are related to the existence of saddle-node bifurcations. Thus, for instance, period- $p$ windows inside chaos arise from a saddle-node of period- $p$ orbits ( $\mathrm{SNp}$ ) and continue with a sequence of period doublings within the corresponding interval. Another example is given by bubbles, which consist in a combination of a SNp with its reverse version.

Periodic oscillations have been studied analytically and numerically by means of different methods and algorithms. Explicit analytical results always complement the exhaustive numerical simulations found in the literature. But, furthermore, they help to understand the effect of the different parameters of the system on the location and characteristics of the orbits. Commonly, period- $p$ points are considered as fixed points of the $p$ th-iterated map. ${ }^{2}$ Thus, period-two (P2) orbits are analyzed via the second-iterated map, period-four (P4) orbits are studied via the fourth-iterated maps, and so on. Since, for example, the fourth recurrence of a scalar quadratic map results in a sixteenth order polynomial, analytical developments based on this approach become almost impracticable rapidly.

To overcome this situation, alternative procedures have been proposed. In Ref. 22, the unknown points are found by inserting the Fourier decomposition of the periodic orbit into the recurrence expressions. Solutions are obtained by reducing difference equations to equivalent polynomials parameterized in terms of an arbitrary variable in Refs. 23 and 24. Approximate representations of the period- $2^{k}(k \geq 1)$ orbits are developed in Ref. 25 by means of a scaling method. In all cases, results deal with particular one- and twodimensional quadratic maps. 


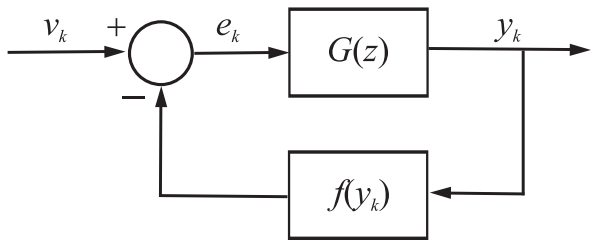

FIG. 1. Input-output representation of discrete-time nonlinear systems.

A FD methodology for the study of orbits emerging from period-doubling and Neimark-Sacker bifurcations in $n$ dimensional maps is presented in Refs. 26-28. Procedures are based on concepts from control theory such as the inputoutput representation of the system, the Fourier decomposition of the orbit, and the closed-loop balance of the involved harmonics. Explicit expressions of the oscillations are obtained by considering a finite Taylor expansion of the involved nonlinearity and a truncated Fourier decomposition of the orbits. Higher-order terms of the balance equations are also neglected by making certain assumptions on the magnitude of the coefficients. These contributions complement Refs. 29-36, demonstrating that the FD approach can be an appropriate option for the analysis of oscillations in continuous-time, discrete-time, and delayed systems.

The aim of this paper is to show the potential of the FD viewpoint to find the analytical and exact solutions of the P4 orbits exhibited by a family of $n$-dimensional maps. Critical conditions corresponding to the onset of the solutions and the general expressions of each periodic point are provided. Developments follow the fundamental ideas of the classical FD method but, in contrast to the previous contributions, the harmonic balance is applied here to the whole finite number of terms constituting the orbit. Moreover, since quadratic nonlinearities are considered, exact Taylor expansions can be used. Solutions are obtained thanks to the application of Gröbner bases for polynomial maps ${ }^{37}$ to the complete set of balance equations. Some results concerning the dynamical characterization of a scalar quadratic map by using these bases can be found in Refs. 38 and 39.

This paper is organized as follows. In Sec. II, preliminary concepts about the FD treatment of $n$-dimensional quadratic maps are introduced. Developments concerning the application of the harmonic balance complemented by Gröbner bases to obtain the analytical expressions of P4 orbits are described in Sec. III. The potentiality of the results is illustrated in Sec. IV by studying a generalized scalar quadratic map and a delayed version of the logistic map. Finally, conclusions are given in Sec. V.

\section{THE FREQUENCY-DOMAIN VIEWPOINT}

Procedures commonly used for the dynamical analysis of maps imply the manipulation of the whole state-variable difference equations of the system. The FD viewpoint considered here is applied to an equivalent input-output representation. As the number of inputs and outputs is normally smaller than the number of internal states, the dimension of the system can be naturally reduced, simplifying the calculations.
The classical Lur'e single-input single-output representation is depicted in Fig. 1, which is composed of a linear dynamical block connected to a nonlinear static one by means of a feedback loop. The variables in this configuration are the input $v_{k} \in \mathbf{R}$, the error $e_{k} \in \mathbf{R}$, and the output $y_{k} \in \mathbf{R}$. Since it is linear, block $G(\cdot)$ is expressed in the domain of the $z$-transformation. The nonlinear block is simply expressed as a smooth function $f(\cdot): \mathbf{R} \rightarrow \mathbf{R}$. As $v_{k}$ is assumed to be zero, the input of $G(\cdot)$ is directly $e_{k}=-f\left(y_{k}\right)$.

This feedback model is sufficiently wide as to represent a great number of nonlinear maps. In particular, let us consider the following $n$-dimensional system:

$$
\begin{aligned}
x_{k+1} & =A x_{k}+B\left(a y_{k}^{2}+b y_{k}+c\right), \\
y_{k} & =C x_{k},
\end{aligned}
$$

with $x_{k} \in \mathbf{R}^{n}, A \in \mathbf{R}^{n \times n}, B \in \mathbf{R}^{n \times 1}, C \in \mathbf{R}^{1 \times n}$, and $a \neq 0$. This map can be seen as a generalization of a family of quadratic maps widely studied in the literature. ${ }^{2,18,20}$ Some representative examples are resumed in Table I.

There exist many distinct but equivalent forms of representing (1) as the block diagram of Fig. 1. In fact, introducing an arbitrary linear term $D y_{k}$, (1) can be rewritten as

$$
\begin{aligned}
x_{k+1} & =A x_{k}+B D C x_{k}+B e_{k}, \\
y_{k} & =C x_{k}, \\
e_{k} & =a y_{k}^{2}+b y_{k}+c-D y_{k} .
\end{aligned}
$$

Then, applying the $z$-transform and making some algebraic operations (see Appendix A for further details), the linear dynamical block $G(\cdot)$ and the function $f(\cdot)$ containing the nonlinearities of the system are

$$
\begin{aligned}
G(z) & =C[z I-(A+B D C)]^{-1} B, \\
f\left(y_{k}\right) & =-\left(a y_{k}^{2}+b y_{k}+c\right) .
\end{aligned}
$$

For simplicity, the linear part of $f(\cdot)$, given by $(b-D) y_{k}$, has been unified as $b y_{k}$. Coefficient $D$ is useful to obtain different realizations but it does not modify the fundamental results. An appropriate election of $D$ corresponds to define a $G(z)$ without zeros and poles at the frequencies involved in the periodic orbit under study $\left(z=e^{i 0}=1, z=e^{i \pi}=-1\right.$ and $z=e^{i \pi / 2}$ ). Notice also that, according to the dimension of matrices $A, B$, and $C$, block $G(z)$ transforms into a scalar rational function in the complex variable $z$. Thus, the analysis of the original $n$-dimensional system is reduced to that of an interconnection between two scalar functions. Inputoutput representations of quadratic maps given in Table I evidence this simplification.

Fixed points $\hat{y}$ of system (3)-(4) can be calculated via the scalar equation $\hat{y}=-G(1) f(\hat{y})=G(1)\left(a \hat{y}^{2}+b \hat{y}+c\right)$, where $G(1)$ is the response of the linear block to a signal of frequency $\omega=0\left(z=e^{i 0}=1\right)$. Then, it is easy to see that

$$
\hat{y}_{ \pm}=\frac{1-b G(1)}{2 a G(1)} \pm \frac{\sqrt{[1-b G(1)]^{2}-4 a c G(1)^{2}}}{2 a G(1)}
$$

which will exist if $\Delta^{2}=[1-b G(1)]^{2}-4 a c G(1)^{2} \geq 0$. The stability of each $\hat{y}_{ \pm}$can be determined by means of the 
TABLE I. Examples of input-output descriptions of some quadratic maps.

I-O representation

\begin{tabular}{|c|c|}
\hline \multicolumn{2}{|l|}{ Scalar quadratic map } \\
\hline$x_{k+1}=x_{k}^{2}+c$ & $\begin{array}{l}A=0, B=C=1, a=1, b=0 \\
G(z)=\frac{1}{z}, f\left(y_{k}\right)=-\left(y_{k}^{2}+c\right) .\end{array}$ \\
\hline Logistic map & \multirow[b]{2}{*}{$\begin{array}{l}A=0, B=C=1, a=-b=-\alpha, c=0 \\
G(z)=\frac{1}{z}, f\left(y_{k}\right)=-\alpha y_{k}\left(1-y_{k}\right)\end{array}$} \\
\hline$x_{k+1}=\alpha x_{k}\left(1-x_{k}\right)$ & \\
\hline $\begin{array}{l}\text { Hénon map } \\
\begin{array}{l}x_{1, k+1}=p x_{2, k}+\rho-x_{1, k}^{2} \\
x_{2, k+1}=x_{1, k}\end{array}\end{array}$ & $\begin{array}{l}A=\left(\begin{array}{cc}0 & p \\
1 & 0\end{array}\right), B^{T}=C=\left(\begin{array}{ll}1 & 0\end{array}\right) \\
a=-1, b=0, c=\rho \\
G(z)=\frac{z}{z^{2}-2 z-p}, f\left(y_{k}\right)=y_{k}^{2}+2 y_{k}-\rho .\end{array}$ \\
\hline $\begin{array}{l}\text { 3D Hénon map } \\
x_{1, k+1}=x_{2, k}+\rho-x_{1, k}^{2} \\
x_{2, k+1}=p x_{1, k}+x_{3, k} \\
x_{3, k+1}=-p x_{1, k}\end{array}$ & $\begin{array}{l}A=\left(\begin{array}{ccc}0 & 1 & 0 \\
p & 0 & 1 \\
-p & 0 & 0\end{array}\right), B^{T}=C=\left(\begin{array}{lll}1 & 0 & 0\end{array}\right), \\
a=-1, b=0, c=\rho \\
G(z)=\frac{z^{2}}{z^{3}-2 z^{2}-p z+p}, f\left(y_{k}\right)=y_{k}^{2}+2 y_{k}-\rho\end{array}$ \\
\hline $\begin{array}{l}\text { Delayed logistic map } \\
x_{k+1}=\alpha x_{k}\left(1-x_{k}\right)-\eta\left(x_{k-\sigma}-x_{k}\right)\end{array}$ & $\begin{array}{l}A=\left(\begin{array}{cccc}0 & \cdots & 0 & -\eta \\
1 & \cdots & 0 & 0 \\
\vdots & \ddots & 0 & 0 \\
0 & \cdots & 1 & 0\end{array}\right), B^{T}=C=\left(\begin{array}{llll}1 & 0 & \cdots & 0\end{array}\right), \\
a=-\alpha, b=\alpha+\eta, c=0 \\
G(z)=\frac{z^{\sigma}}{z^{\sigma+1}+\eta}, f\left(y_{k}\right)=\alpha y_{k}^{2}-(\alpha+\eta) y_{k}\end{array}$ \\
\hline
\end{tabular}

open-loop function $G(z) J$, where $J$ is the derivative of $f(\cdot)$ at $\hat{y}_{ \pm}$. In particular, the fixed point will be non hyperbolic if $G\left(e^{i \omega}\right) J=-1+i 0$ for certain $\omega=\omega_{o}$. Thus, for instance, period-doubling bifurcations occur if $\omega_{o}=\pi$, while NeimarkSacker bifurcations occur if $\omega_{o}=\omega_{H}$, with the exception of $\omega_{H}$ values such that $e^{i n \omega_{H}}=1$ for $n=1,2,3$, or 4 since they correspond to the case of strong resonances $1: 1,1: 2,1: 3$, and $1: 4$, respectively. Details about stability analysis of the resulting bifurcations can be found in Refs. 26-28.

The input-output representation facilitates the application of the harmonic balance method for the characterization of the orbits. To do that, the oscillation needs to be represented by means of an appropriate Fourier series decomposition. In the case of continuous-time nonlinear systems, the Fourier series is truncated up to a determined number of terms since the orbit decomposition usually contains infinite harmonics. This is also accompanied by considering a finite Taylor expansion of the nonlinear function in order to obtain a finite number of equations to be solved. So, the harmonic balance certainly provides an approximation of the actual solution. $^{29-33}$

Discrete-time nonlinear systems can undergo periodic or quasiperiodic orbits. In the last case, an infinite number of terms is required to represent the oscillation accurately. So, analogously to the continuous-time counterpart, the harmonic balance method necessarily implies neglecting higher harmonic terms, leading to approximate orbit representations. ${ }^{26,28}$ However, if the orbit is periodic with period $p=2 \pi / \omega$ $\left(p \in \mathbf{Z}_{+}\right.$or even $\mathbf{Q}_{+}$) such as $y_{k}=y_{k+p}$, the complex exponentials $e^{i r \omega k}(r \in \mathbf{N})$ conforming the Fourier series repeat if a harmonic number greater than $p$ is considered. In fact, if $r=p+1$, then $e^{i(p+1) \omega k}=e^{i(2 \pi+\omega) k}=e^{i \omega k}$ corresponding to $r=1$ and, in general, if $r \geq p$, then $e^{i r \omega k}=e^{i \tilde{r} \omega k}$, where $\tilde{r}$ stands for the value of $r$ modulus $p$. Therefore, only a finite number of harmonics are needed to represent exactly a period- $p$ oscillation. Now, if the Taylor expansion of the nonlinear function is truncated, results will be approximate as well, as in Ref. 27. Since quadratic functions are considered here, higher-order terms are naturally zero making possible to provide analytic and exact solutions of the periodic orbits.

\section{ANALYTICAL PERIODIC SOLUTIONS OF QUADRATIC MAPS}

In this section, the exact solutions of the P2 and P4 orbits exhibited by quadratic map (1) are obtained. As could be expected, coefficients involved in the Fourier decomposition of the P2 orbits are calculated almost effortlessly. ${ }^{40}$ The same does not occur in the case of the P4 oscillations. The harmonic balance results in a set of four quadratic polynomials depending on four unknown variables. The complexity is overcame by using an algebraic tool known as Gröbner bases. ${ }^{37}$

\section{A. Period-two solutions}

The Fourier decomposition of a P2 orbit can be expressed as

$$
y_{k}=\hat{y}+Y_{0}+Y_{1} e^{i \pi k},
$$

where $\hat{y}=\hat{y}_{+}$or $\hat{y}_{-}, Y_{0} \in \mathbf{R}$ is a correction of the fixed point $\hat{y}$ to achieve the center or average value $\tilde{Y}_{0}=\hat{y}+Y_{0}$ of the oscillation and $Y_{1} \in \mathbf{R}$ represents the amplitude of the main 
harmonic term ( $e^{-i \pi k}$ is omitted since it is equivalent to $\left.e^{i \pi k}\right)$. Thus, the two points conforming the discrete oscillation are given by

$$
\begin{aligned}
& y_{P 2,1}=\hat{y}+Y_{0}-Y_{1}, \\
& y_{P 2,2}=\hat{y}+Y_{0}+Y_{1} .
\end{aligned}
$$

As $y_{k}$ is the input of the nonlinear block, the respective output $f\left(y_{k}\right)$ can be calculated. For simplicity, $f(\cdot)$ is written by means of its exact Taylor series expansion around the fixed point $\hat{y}$, i.e.,

$$
f\left(y_{k}\right)=f(\hat{y})+J\left(y_{k}-\hat{y}\right)+K\left(y_{k}-\hat{y}\right)^{2},
$$

with $J=D f(\hat{y})=-(2 a \hat{y}+b)$ and $K=1 / 2 D^{2} f(\hat{y})=-a$. Then, replacing $y_{k}$ by (5) and making some routine algebraic manipulations, it is obtained

$$
f\left(y_{k}\right)=f(\hat{y})+F_{0}+F_{1} e^{i \pi k}
$$

with

$$
\begin{aligned}
& F_{0}=J Y_{0}+K\left(Y_{0}^{2}+Y_{1}^{2}\right), \\
& F_{1}=J Y_{1}+2 K Y_{0} Y_{1} .
\end{aligned}
$$

Since $e_{k}=-f\left(y_{k}\right)$ is the input of the linear block $G(\cdot)$ and it preserves the harmonic decomposition of the original $y_{k}$, the feedback loop of Fig. 1 is closed by establishing the harmonic balance

$$
\begin{aligned}
& Y_{0}=-G(1) F_{0}, \\
& Y_{1}=-G(-1) F_{1},
\end{aligned}
$$

where $G(-1)$ is the response of $G(\cdot)$ to the harmonic frequency $\omega=\pi\left(z=e^{i \pi}=-1\right)$. The derivation of these equations is included in Appendix B.

Now, combining (7)-(10), a set of two quadratic polynomials in the real variables $Y_{0}$ and $Y_{1}$ is obtained. Thus,

$$
\begin{aligned}
& 0=[1+G(1) J] Y_{0}+G(1) K\left(Y_{0}^{2}+Y_{1}^{2}\right), \\
& 0=[1+G(-1) J] Y_{1}+2 G(-1) K Y_{0} Y_{1} .
\end{aligned}
$$

Omitting the existence of 1:1 and 1:2 resonances and assuming the period-doubling critical condition has been already verified, it can be affirmed that $G(1) J \neq-1+i 0$ and $G(-1) J \neq-1+i 0$ for all parameter combination corresponding to the occurrence of a $\mathrm{P} 2$ orbit. Then, replacing Jacobian $J$ by its expression as a function of $a, b$, and $c$, Fourier coefficients representing the $\mathrm{P} 2$ orbit of a quadratic map are obtained. The two possible $Y_{1}$ solutions have the same absolute value but opposite signs. As both correspond to Fourier decompositions of the same orbit but starting from different initial points $\left(y_{P 2,1}\right.$ or $\left.y_{P 2,2}\right)$, the positive $Y_{1}$ solution is only reported here. Simplifying the notation as $G_{1}=G(1)$ and $G_{-1}=G(-1)$

$$
\tilde{Y}_{0}=\hat{y}+Y_{0}=\frac{1-b G_{-1}}{2 a G_{-1}},
$$

$$
Y_{1}=\frac{\sqrt{2 G_{-1}\left(1-b G_{-1}\right)+G_{1}\left[\left(b^{2}-4 a c\right) G_{-1}^{2}-1\right]}}{2 a \sqrt{G_{1}} G_{-1}} .
$$

Notice that $Y_{1}$ will be a real value whenever $\Delta_{2}^{2}=2 G_{-1}\left[1-b G_{-1}\right]+G_{1}\left[\left(b^{2}-4 a c\right) G_{-1}^{2}-1\right] \geq 0$, which coincides with the critical condition for the appearance of period-doubling bifurcations $[G(-1) J=-1+i 0]$ but expressed as a function of the parameters of the system.

\section{B. Period-four solutions}

To maintain the structure of the previous decomposition, the Fourier series representing a P4 orbit is ordered as

$$
y_{k}=\hat{y}+Y_{0}+Y_{1} e^{i \pi k}+Y_{2} e^{i \frac{\pi}{2} k}+\bar{Y}_{2} e^{-i \frac{\pi}{2} k},
$$

where "-" stands for the conjugate operator, $Y_{0} \in \mathbf{R}$ can be seen again as a correction to achieve the average value of the oscillation and $Y_{1} \in \mathbf{R}$ and $Y_{2}=Y_{2 R}+i Y_{2 I} \in \mathbf{C}$ are the coefficients of the rest of harmonics ( $e^{-i \frac{\pi}{2} k}$ is equivalent to $\left.e^{i \frac{3 \pi}{2} k}\right)$. Thus, periodic points can be recast as

$$
\begin{aligned}
& y_{P 4,1}=\hat{y}+Y_{0}-Y_{1}-2 Y_{2 I}, \\
& y_{P 4,2}=\hat{y}+Y_{0}+Y_{1}-2 Y_{2 R}, \\
& y_{P 4,3}=\hat{y}+Y_{0}-Y_{1}+2 Y_{2 I}, \\
& y_{P 4,4}=\hat{y}+Y_{0}+Y_{1}+2 Y_{2 R} .
\end{aligned}
$$

Besides solution (13) could be thought as an expansion of (5), coefficients $Y_{0}$ and $Y_{1}$ are not necessarily the same. They will coincide at the onset of $\mathrm{P} 4$ orbits $\left(Y_{2}=0\right)$, which are preceded by $\mathrm{P} 2$ solutions.

Considering that (13) is the input of the nonlinear block, the output of the Taylor expansion (6) results in

$$
\begin{aligned}
f\left(y_{k}\right)= & f(\hat{y})+J\left(Y_{0}+Y_{1} e^{i \pi k}+Y_{2} e^{i \frac{\pi}{2} k}+\bar{Y}_{2} e^{-i \frac{\pi}{2} k}\right) \\
& +K\left[Y_{0}^{2}+Y_{1}^{2}+2\left|Y_{2}\right|^{2}+\left(2 Y_{0} Y_{1}+Y_{2}^{2}+\bar{Y}_{2}^{2}\right) e^{i \pi k}\right. \\
& \left.+2\left(Y_{0} Y_{2}+Y_{1} \bar{Y}_{2}\right) e^{i \frac{\pi}{2} k}+2\left(Y_{0} \bar{Y}_{2}+Y_{1} Y_{2}\right) e^{-i \frac{\pi}{2} k}\right],
\end{aligned}
$$

which is equivalent to

$$
f\left(y_{k}\right)=f(\hat{y})+F_{0}+F_{1} e^{i \pi k}+F_{2} e^{i \frac{\pi}{2} k}+\bar{F}_{2} e^{-i \frac{\pi}{2} k},
$$

with

$$
\begin{aligned}
& F_{0}=J Y_{0}+K\left(Y_{0}^{2}+Y_{1}^{2}+2\left|Y_{2}\right|^{2}\right), \\
& F_{1}=J Y_{1}+K\left(2 Y_{0} Y_{1}+Y_{2}^{2}+\bar{Y}_{2}^{2}\right), \\
& F_{2}=J Y_{2}+2 K\left(Y_{0} Y_{2}+Y_{1} \bar{Y}_{2}\right) .
\end{aligned}
$$

Then, the harmonic balance originated by closing the loop in Fig. 1 (see Appendix B for a detailed justification) is given by

$$
\begin{aligned}
& Y_{0}=-G(1) F_{0}, \\
& Y_{1}=-G(-1) F_{1}, \\
& Y_{2}=-G(i) F_{2},
\end{aligned}
$$

where $G(i) \in \mathbf{C}$ is the response of $G(\cdot)$ to a signal of frequency $\omega=\pi / 2\left(z=e^{i \pi / 2}=i\right)$. 
The combination of (14)-(19) leads to three polynomials in variables $Y_{0}, Y_{1}$, and $Y_{2}$ with complex coefficients. To facilitate the calculations, balance equations are transformed into four real polynomials in $Y_{0}, Y_{1}, Y_{2 R}$, and $Y_{2 I}$. Using the notation $G_{1}=G(1), G_{-1}=G(-1), \quad$ and $\quad G_{i}=G(i)=$ $G_{i R}+i G_{i I}$ with $G_{i I} \neq 0$ (since the critical condition of resonance 1:4 is omitted), they are expressed as

$$
\begin{aligned}
0= & \left(1+G_{1} J\right) Y_{0}+G_{1} K\left(Y_{0}^{2}+Y_{1}^{2}+2 Y_{2 R}^{2}+2 Y_{2 I}^{2}\right), \\
0= & \left(1+G_{-1} J\right) Y_{1}+2 G_{-1} K\left(Y_{0} Y_{1}+Y_{2 R}^{2}-Y_{2 I}^{2}\right), \\
0= & \left\{1+G_{i R}\left[J+2 K\left(Y_{0}+Y_{1}\right)\right]\right\} Y_{2 R} \\
& -G_{i I}\left[J+2 K\left(Y_{0}-Y_{1}\right)\right] Y_{2 I}, \\
0= & G_{i I}\left[J+2 K\left(Y_{0}+Y_{1}\right)\right] Y_{2 R} \\
& +\left\{1+G_{i R}\left[J+2 K\left(Y_{0}-Y_{1}\right)\right]\right\} Y_{2 I} .
\end{aligned}
$$

From the last two equations, it can be deduced that the $Y_{2}$ solution will be different from zero whenever

$$
\left|\begin{array}{cc}
1+G_{i R} \Gamma_{+} & -G_{i I} \Gamma_{-} \\
G_{i I} \Gamma_{+} & 1+G_{i R} \Gamma_{-}
\end{array}\right|=0
$$

where $\Gamma_{ \pm}=\left[J+2 K\left(Y_{0} \pm Y_{1}\right)\right]$. Notice that if $Y_{0}$ and $Y_{1}$ are replaced by the values corresponding to preceding dynamics such as fixed points or P2 orbits (as it occurs in a perioddoubling cascade), this determinant reduces to the critical condition for the onset of two possible scenarios involving $\mathrm{P} 4$ orbits in a $n$-dimensional map.

- P4 orbits emerging from a fixed point. As coefficients $Y_{0}$ and $Y_{1}$ are equal to zero, the determinant transforms into the critical condition

$$
0=G_{1}^{2}-2 G_{1} G_{i R}+2\left(G_{1} G_{i R}-\left|G_{i}\right|^{2}\right) \Delta+\left|G_{i}\right|^{2} \Delta^{2} .
$$

- P4 orbits emerging from a P2 oscillation. Substituting $Y_{0}$ and $Y_{1}$ by (11) and (12), respectively, condition (20) results equivalent to

$$
0=\left|G_{i}\right|^{2}\left(G_{1}-\Delta_{2}^{2}\right)-2 G_{1} G_{-1} G_{i R}+G_{1} G_{-1}^{2} .
$$

Assuming that (20) is verified, coefficients of the Fourier series representing P4 orbits can be obtained. To simplify the algebraic manipulation of the balance equations, $J$ and $K$ are replaced by its expressions as a function of the parameters and $Y_{0}$ is written as $\tilde{Y}_{0}-\hat{y}$. Thus, a set of polynomials $P=$ $\left\{p_{1}, p_{2}, p_{3}, p_{4}\right\}$ with

$$
\begin{aligned}
p_{1}= & \left(1-b G_{1}\right) \tilde{Y}_{0}-a G_{1} \tilde{Y}_{0}^{2}-a G_{1} Y_{1}^{2}-2 a G_{1} Y_{2 R}^{2} \\
& -2 a G_{1} Y_{2 I}^{2}-c G_{1}, \\
p_{2}= & \left(1-b G_{-1}\right) Y_{1}-2 a G_{-1} \tilde{Y}_{0} Y_{1}-2 a G_{-1} Y_{2 R}^{2}+2 a G_{-1} Y_{2 I}^{2}, \\
p_{3}= & \left(1-b G_{i R}\right) Y_{2 R}-2 a G_{i R} \tilde{Y}_{0} Y_{2 R}-2 a G_{i R} Y_{1} Y_{2 R}+b G_{i I} Y_{2 I} \\
& +2 a G_{i I} \tilde{Y}_{0} Y_{2 I}-2 a G_{i I} Y_{1} Y_{2 I}, \\
p_{4}= & \left(1-b G_{i R}\right) Y_{2 I}-2 a G_{i R} \tilde{Y}_{0} Y_{2 I}+2 a G_{i R} Y_{1} Y_{2 I}-b G_{i I} Y_{2 R} \\
& -2 a G_{i I} \tilde{Y}_{0} Y_{2 R}-2 a G_{i I} Y_{1} Y_{2 R},
\end{aligned}
$$

is defined. Due to the involved complexity, the real roots of $P$ are found by using Gröbner bases. This is an algebraic mechanism, equivalent to the Gauss triangulation for linear equations, that permits to obtain a new polynomial set containing the same roots of $P$. Since the algorithm contemplates the variable elimination following a determined monomial order, the equivalent polynomial system results simpler to be solved. ${ }^{37}$

So, let us suppose that $P$ generates the ideal ${ }^{41} I$ belonging to the polynomial ring in variables $Y_{2 R}, Y_{2 I}, Y_{1}, \tilde{Y}_{0}$ with coefficients in the field of the rational functions of the parameters. Setting the lexicographical order $Y_{2 R} \succ Y_{2 I} \succ Y_{1}$ $\succ \tilde{Y}_{0}$, the $r$ th elimination ideal $I_{r}$ contains all the consequences of equations $p_{1}=p_{2}=p_{3}=p_{4}=0$, which eliminate the first $r$ variables, with $r=1,2$, and 3 . The sequence of ideals $I \supset I_{1} \supset I_{2} \supset I_{3}$ leads to the elimination of $Y_{2 R}, Y_{2 I}$, and $Y_{1}$. The last ideal consists of polynomials in the last variable $\tilde{Y}_{0}$ alone. Algorithms for computing Gröbner bases are implemented in many commercial or academic computer programs such as Maple, Mathematica, and Magma. ${ }^{37,39}$ When the lexicographical order is considered, the calculation of these bases provides automatically a basis of all ideals $I_{r}$. In this case, the obtained Gröbner basis is composed of six polynomials. One of them, denoted here as $g$, corresponds to the basis of $I_{3}$ and then it only depends on the unknown $\tilde{Y}_{0}$. This particular polynomial makes easier to find the desired solutions of the system.

Making algebraic manipulations, $g$ can be factorized into a product of distinct irreducible polynomials as $g=g_{1} g_{2} g_{3}$, where

$$
\begin{aligned}
& g_{1}=\left(1-b G_{1}\right) \tilde{Y}_{0}-a G_{1} \tilde{Y}_{0}^{2}-c G_{1}, \\
& g_{2}=1-b G_{-1}-2 a G_{-1} \tilde{Y}_{0}, \\
& g_{3}=G_{1} G_{-1}\left(G_{i R}+2 b G_{i I}^{2}\right)+G_{1}\left|G_{i}\right|^{2}\left[2-5 b G_{-1}-b\left|G_{i}\right|^{2}\right. \\
& \times\left(3 b^{2} G_{-1}-2 b+4 a c G_{-1}\right) \\
& \left.+G_{i R}\left(7 b^{2} G_{-1}-4 b+4 a c G_{-1}\right)\right] \\
& -\left\{4 a\left|G_{i}\right|^{2}\left(G_{i R}-b\left|G_{i}\right|^{2}\right) G_{-1}+2 a G_{1}\left[\left(5 G_{i R}^{2}+3 G_{i I}^{2}\right) G_{-1}\right.\right. \\
& +4 G_{i R}\left|G_{i}\right|^{2}\left(1-4 b G_{-1}\right)+\left|G_{i}\right|^{4}\left(11 b^{2} G_{-1}-4 b\right. \\
& \left.\left.\left.+4 a c G_{-1}\right)\right]\right\} \tilde{Y}_{0}+8 a^{2}\left|G_{i}\right|^{2}\left[4 G_{1} G_{i R} G_{-1}\right. \\
& \left.+\left|G_{i}\right|^{2}\left(G_{1}+G_{-1}-6 b G_{1} G_{-1}\right)\right] \tilde{Y}_{0}^{2}-32 a^{3} G_{1}\left|G_{i}\right|^{4} G_{-1} \tilde{Y}_{0}^{3} .
\end{aligned}
$$

The roots of each $g_{i}(i=1,2,3)$ are related to three different solutions of the map:

- Fixed points: The quadratic roots of factor $g_{1}$ conform the fixed points of the map as they are solutions of $P=0$ for $Y_{1}=Y_{2 R}=Y_{2 I}=0$.

- Period-two points: The unique root of factor $g_{2}$ is part of the period-two solution as it verifies $P=0$ together with $0=\left(1-b G_{-1}\right) Y_{1}-2 a G_{-1} \tilde{Y}_{0} Y_{1} \quad$ and $\quad Y_{2 R}=Y_{2 I}=0$. These expressions are equivalent to coefficients (11) and (12) given in Section III A.

- Period-four points: The roots of factor $g_{3}$ correspond to P4 orbits since they are solutions of $P=0$ for $Y_{2 R} \neq 0$ and $Y_{2 I} \neq 0$. 
TABLE II. Solutions of the scalar quadratic maps.

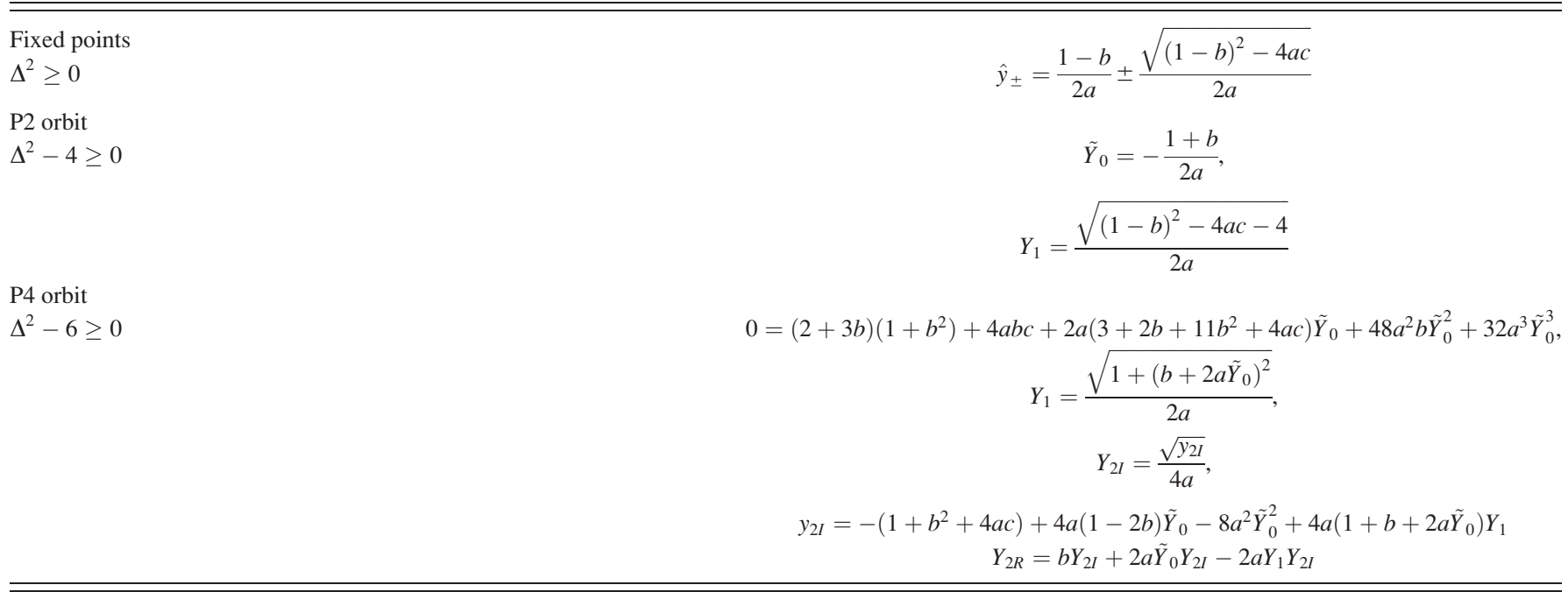

Since $g_{3}$ is a cubic polynomial, it will always have a real root. According to the parameter values, this solution could be the only one (since the other two are conjugate complex roots) or be part of a set of three real roots. The former case is associated with the appearance of a unique P4 orbit. Critical condition (22) indicates the onset of this oscillation when it arises from a previous $\mathrm{P} 2$ orbit. The latter one is connected to the occurrence of three $\mathrm{P} 4$ orbits, with their respective stability, simultaneously. This can describe, for example, the classical scenario of $\mathrm{P} 4$ windows after the onset of chaos or, as it will be shown in Sec. IV B, the bistability phenomenon developed by delayed maps. The final number of P4 solutions is actually determined by the existence of real roots for the rest of the coefficients $\left(Y_{1}, Y_{2 R}\right.$, and $\left.Y_{2 I}\right)$.

To only find the expressions of the Fourier coefficients representing any of the three possible P4 solutions, factor $g_{3}$ is added to the original set of harmonic balance polynomials generating a new $\tilde{P}=P \cup g_{3}$. Applying now Gröbner bases to $\tilde{P}$, the desired four polynomials with triangular structure are obtained. One of them is precisely $g_{3}$, which gives the three possible $\tilde{Y}_{0}$ solutions. The rest lead to the calculation of $Y_{1}, Y_{2 R}$, and $Y_{2 I}$ recursively. The complete set is given by

$$
\begin{aligned}
0= & G_{1} G_{-1}\left(G_{i R}+2 b G_{i I}^{2}\right)+G_{1}\left|G_{i}\right|^{2}\left[2-5 b G_{-1}-b\left|G_{i}\right|^{2}\left(3 b^{2} G_{-1}-2 b+4 a c G_{-1}\right)+G_{i R}\left(7 b^{2} G_{-1}-4 b+4 a c G_{-1}\right)\right] \\
& -\left\{2 a G_{1}\left[\left(5 G_{i R}^{2}+3 G_{i I}^{2}\right) G_{-1}+4 G_{i R}\left|G_{i}\right|^{2}\left(1-4 b G_{-1}\right)+\left|G_{i}\right|^{4}\left(11 b^{2} G_{-1}-4 b+4 a c G_{-1}\right)\right]\right. \\
& \left.+4 a\left|G_{i}\right|^{2}\left(G_{i R}-b\left|G_{i}\right|^{2}\right) G_{-1}\right\} \tilde{Y}_{0}+8 a^{2}\left|G_{i}\right|^{2}\left[4 G_{1} G_{i R} G_{-1}+\left|G_{i}\right|^{2}\left(G_{1}+G_{-1}-6 b G_{1} G_{-1}\right)\right] \tilde{Y}_{0}^{2}-32 a^{3} G_{1}\left|G_{i}\right|^{4} G_{-1} \tilde{Y}_{0}^{3} \\
0= & 1-2 b G_{i R}+b^{2}\left|G_{i}\right|^{2}-4 a\left(G_{i R}-b\left|G_{i}\right|^{2}\right) \tilde{Y}_{0}+4 a^{2}\left|G_{i}\right|^{2} \tilde{Y}_{0}^{2}-4 a^{2}\left|G_{i}\right|^{2} Y_{1}^{2} \\
0= & G_{1}\left[1-2 b G_{i R}+\left(b^{2}+4 a c\right)\left|G_{i}\right|^{2}\right] G_{-1}-4\left[a G_{1} G_{i R}+\left(a-2 a b G_{1}\right)\left|G_{i}\right|^{2}\right] G_{-1} \tilde{Y}_{0}+8 a^{2} G_{1}\left|G_{i}\right|^{2} G_{-1} \tilde{Y}_{0}^{2} \\
& +4 a G_{1}\left|G_{i}\right|^{2}\left(1-b G_{-1}\right) Y_{1}-8 a^{2} G_{1}\left|G_{i}\right|^{2} G_{-1} \tilde{Y}_{0} Y_{1}+16 a^{2} G_{1}\left|G_{i}\right|^{2} G_{-1} Y_{2 I}^{2}, \\
0= & \left(G_{i R}-b\left|G_{i}\right|^{2}\right) Y_{2 I}-2 a\left|G_{i}\right|^{2} \tilde{Y}_{0}+2 a\left|G_{i}\right|^{2} Y_{1} Y_{2 I}-G_{i I} Y_{2 R},
\end{aligned}
$$

where the main coefficient of each polynomial is nonzero since the critical condition of resonance $1: 4$ is omitted $\left(G_{i I} \neq 0\right)$ and the feedback representation of the map is defined assuring that $G_{1} \neq 0, G_{-1} \neq 0$ and $\left|G_{i}\right|^{2} \neq 0$. As can be observed, there exist two possible $Y_{1}$ values for each $\tilde{Y}_{0}$ solution; and also, two $Y_{2}=Y_{2 R}+i Y_{2 I}$ for each pair $\tilde{Y}_{0}-Y_{1}$. These four solutions actually correspond to four equivalent Fourier decompositions of the same orbit. The difference between them lies in the initial point $\left(y_{P 4, i}\right.$ with $\left.i=1, \ldots, 4\right)$ selected to reproduce the periodic sequence.

It is worth mentioning that a similar procedure can be followed by considering factors $g_{1}$ and $g_{2}$. Obtained solutions will coincide with the expressions of the fixed points and the P2 orbits given in Sections II and III A.

\section{EXAMPLES}

In this section, analytical results are used to describe the different P4 solutions exhibited by a generalized scalar map and a delayed logistic map. In the second case, the dimension of the complete state-variable model depends on the delay in time adopted by the control law. The equivalent input-output representation makes it possible to reduce the dimension of the states, facilitating the calculations. ${ }^{42}$

\section{A. Scalar maps}

As inferred from Table I, the input-output representation of the whole family of scalar quadratic maps can be generalized as $G(z)=1 / z$ and $f\left(y_{k}\right)=-\left(a y_{k}^{2}+b y_{k}+c\right)$ with 


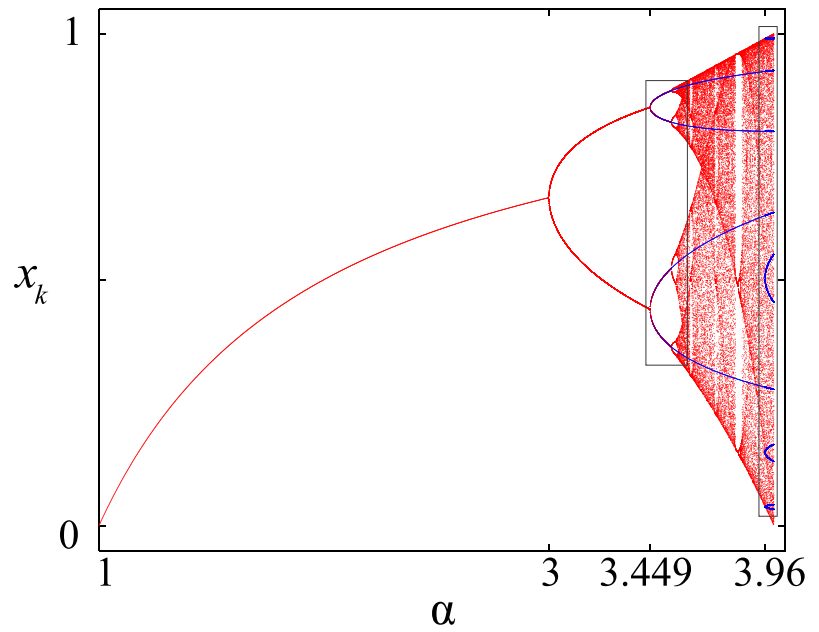

FIG. 2. Period-doubling cascade developed by the logistic map as the grow rate is increased. Red: numerical simulations; blue: exact solutions obtained by the FD methodology.

$y_{k} \in \mathbf{R}$. Since $G_{1}=1, G_{-1}=-1, G_{i}=-i$, and also $\Delta^{2}=(1-b)^{2}-4 a c$, analytical expressions simplify greatly, leading to the results resumed in Table II. Fixed points are defined whenever $\Delta^{2} \geq 0$ while P2 orbits occur for $\Delta^{2}-4 \geq 0$. In the case of $\mathrm{P} 4$ points, critical conditions (21) and (22) were analyzed. As expected, there do not exist real $a, b$, and $c$ combinations that verify condition (21) corresponding to the appearance of $\mathrm{P} 4$ orbits directly from the fixed point. According to (22), P4 orbits emerge from the preceding $\mathrm{P} 2$ solutions at $\Delta^{2}-6=0$.

The study of the number of real roots of the cubic polynomial in $\tilde{Y}_{0}$ (see Table II) permits to complete the dynamical scenario. Analyzing the coefficients of the polynomial and calculating the respective discriminant, ${ }^{43}$ it can be determined that (i) for $6 \leq \Delta^{2}<4+3 \cdot 2^{2 / 3}$, there is one real root, which is equivalent to the unique P4 orbit; (ii) for $\Delta^{2}=4+3 \cdot 2^{2 / 3}$, there are three real roots of which two are equal, indicating that a SN4 adds to the previous solution; (iii) for $\Delta^{2}>4+3 \cdot 2^{2 / 3}$, there are three real and unequal roots, validating that three different $\mathrm{P} 4$ orbits coexist in the

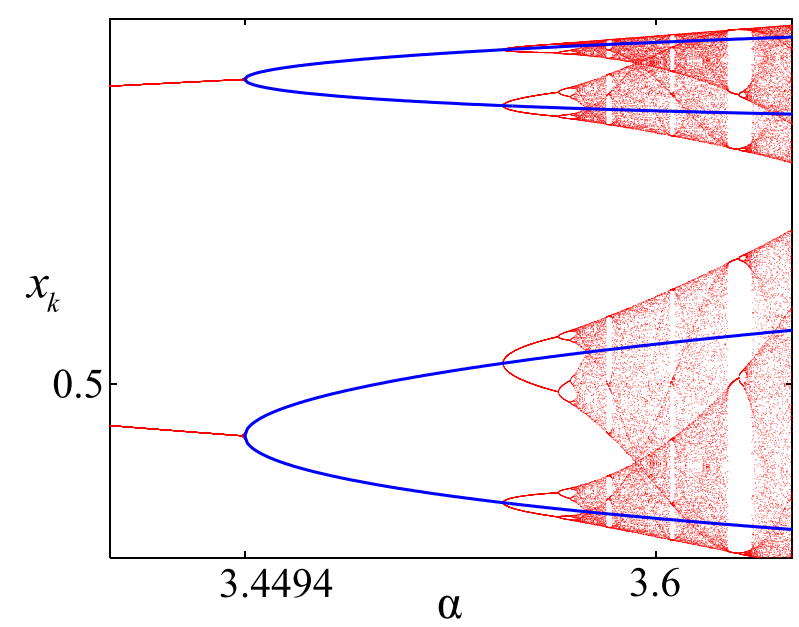

FIG. 3. Blow-up of the P4 branches within the period-doubling cascade. Red: numerical simulations; blue: exact solutions obtained by the FD methodology.

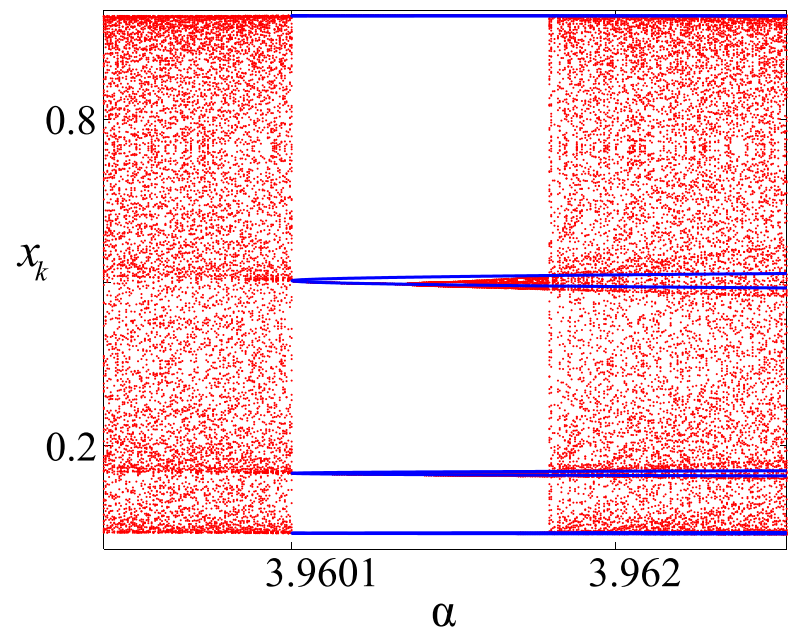

FIG. 4. Blow-up of the P4 window inside chaos. Red: numerical simulations; blue: exact solutions obtained by the FD methodology.

system. Indeed, condition (ii) corresponds to the creation of a $\mathrm{P} 4$ window in the middle of chaos.

The logistic ${ }^{1,2,16,20}$ map $x_{k+1}=\alpha x_{k}\left(1-x_{k}\right)$ with growth rate $\alpha \geq 1$ is chosen to illustrate these results numerically. In this case, $a=-b=-\alpha$ and $c=0$ so that there exists a unique nontrivial fixed point $\hat{y}_{-}=1-1 / \alpha$, which is stable for $1 \leq \alpha \leq 3$ ( $\hat{y}_{+}=0$ is always unstable). Based on Table II, P2 and P4 orbits belonging to the period-doubling cascade emerge for $\alpha \geq 3$ and $\alpha \geq \alpha_{P 4}=1+\sqrt{6}$, respectively. Once the map becomes chaotic, the P4 window occurs at $\alpha=\alpha_{S N 4}=1+\sqrt{4+3 \cdot 2^{2 / 3}}$. Figure 2 shows the complete route to chaos (red) of the logistic map as parameter $\alpha$ is increased. The exact (stable and unstable) P4 solutions provided by expressions in Table II have been overhead (blue). Period-four branches within the cascade are plotted in detail in Fig. 3. The blow-up of the P4 window inside chaos can be seen in Fig. 4. In both figures, there exists a complete coincidence between analytical and numerical results.

\section{B. Delayed logistic map}

In the chaos control technique proposed by Pyragas, ${ }^{44}$ unstable periodic orbits are stabilized by using time-delayed versions of the states in the feedback loop. ${ }^{45}$ Besides its simplicity, this controller can provoke that new unexpected attractors coexist with the desired dynamics giving rise to the so-called bistability phenomenon. ${ }^{11-13}$ Developments of Sec. III help to understand how the Pyragas method affects the characteristics of the orbits with period up to four exhibited by the logistic map. Analytical results concerning small delay values can be found in the literature. ${ }^{13,46}$ For larger delays, nonlinear dynamics are studied by means of numerical calculations.

The delayed version of the logistic map can be expressed as $x_{k+1}=\alpha x_{k}\left(1-x_{k}\right)-\eta\left(x_{k-\sigma}-x_{k}\right)$, where $\alpha$ is the growth rate of the original system $(\alpha \geq 1), \eta$ is the control gain $(-1<\eta<1)$, and $\sigma$ is the delay in time $(\sigma \geq 1)$. The feedback loop implies an increase in the dimension of the state-variable system. This extension is overcame here by studying the map via the FD viewpoint. In fact, the 
TABLE III. Period-two solutions of the delayed logistic map.

\begin{tabular}{lcc}
\hline \hline Parity & Criticality & Solutions \\
\hline Even & $\alpha-3=0$ & $\tilde{Y}_{0}=\frac{1+\alpha}{2 \alpha}$ \\
& $\alpha-3-2 \eta=0$ & $Y_{1}=\frac{\sqrt{(\alpha-3)(\alpha+1)}}{2 \alpha}$ \\
Odd & $\tilde{Y}_{0}=\frac{1+\alpha+2 \eta}{2 \alpha}$ \\
& & $Y_{1}=\frac{\sqrt{(\alpha-3-2 \eta)(\alpha+1+2 \eta)}}{2 \alpha}$ \\
\hline \hline
\end{tabular}

equivalent input-output representation is given by $G(z)=$ $z^{\sigma} /\left(z^{\sigma+1}+\eta\right)$ and $f\left(y_{k}\right)=\alpha y_{k}^{2}-(\alpha+\eta) y_{k}$ independently of the arbitrary delay value (Table I).

As it is known, the Pyragas control maintains the fixed points of the original map $(\eta=0)$, i.e., $\hat{y}_{-}=1-1 / \alpha$ and $\hat{y}_{+}=0$. However, the posterior dynamical behavior and the appearance of new attractors will depend on the gain and delay of the feedback loop.

- Period-two solutions: Block $G(\cdot)$ evaluated at $\omega=\pi$ gives $G_{-1}=(-1)^{\sigma} /\left[(-1)^{\sigma+1}+\eta\right]$, suggesting that the $\sigma$ parity influences in the way the control changes the P2 orbits. Considering that $G_{1}=1 /(1+\eta)$ and

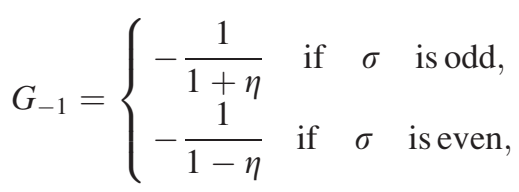

the two possible solutions derived from the expressions of Section III A are resumed in Table III. As can be inferred, P2 orbits are equal to those developed by the scalar map $(\eta=0)$ for even $\sigma$ values. However, they differ from the original ones for odd $\sigma$ values. In particular, the onset of P2 solutions is shifted to higher (lower) $\alpha$ values for gain $\eta>0(\eta<0)$, increasing (decreasing) the interval where $\hat{y}_{-}$is stable.

- Period-four solutions: In this case, it results $G_{i}=e^{i \sigma \frac{\pi}{2}} /\left(e^{i(\sigma+1) \frac{\pi}{2}}+\eta\right)$. Since $\omega=\pi / 2$ conforms the complex fourth roots of unity and also $e^{i \sigma \frac{\pi}{2}}=e^{i(\tilde{\sigma}+4 s) \frac{\pi}{2}}=$ $e^{i \tilde{\sigma} \frac{\pi}{2}}$ with $\tilde{\sigma}=0,1,2,3$ and $s \in \mathbf{Z}, G_{i}$ presents four types of dynamical responses. For even $\sigma$ values, $G_{i I}=-1 /(1+$ $\left.\eta^{2}\right)$ while

$$
G_{i R}=\left\{\begin{array}{lll}
\frac{\eta}{1+\eta^{2}} & \text { if } & \sigma \equiv 0(\bmod 4), \\
-\frac{\eta}{1+\eta^{2}} & \text { if } & \sigma \equiv 2(\bmod 4)
\end{array}\right.
$$

For odd $\sigma$ values, $G_{i R}=0$ while

$$
G_{i I}=\left\{\begin{array}{lll}
-\frac{1}{1-\eta} & \text { if } & \sigma \equiv 1(\bmod 4), \\
-\frac{1}{1+\eta} & \text { if } & \sigma \equiv 3(\bmod 4) .
\end{array}\right.
$$

From (21), it is found that the delayed logistic map does not develop P4 points emerging from a fixed point. Condition (22) permits to affirm that there exist P4 orbits
TABLE IV. Critical conditions for the onset of P4 branches within the period-doubling cascade developed by the delayed logistic map.

\begin{tabular}{lc}
\hline \hline Delay classes $(\bmod 4)$ & Critical condition \\
\hline$\sigma \equiv 0$ & $\alpha-1-\sqrt{6}=0$ \\
$\sigma \equiv 1$ & $\alpha-1-\sqrt{6+2 \eta(4+3 \eta)}=0$ \\
$\sigma \equiv 2$ & $\alpha-1-\sqrt{6-4 \eta(1-\eta)}=0$ \\
$\sigma \equiv 3$ & $\alpha-1-\sqrt{6}(1+\eta)=0$ \\
\hline \hline
\end{tabular}

induced by $\mathrm{P} 2$ oscillations but their occurrence depends on the congruence class of delay $\sigma$, as listed in Table IV. These $\mathrm{P} 4$ solutions are related to the independent real root of the cubic polynomial in $\tilde{Y}_{0}$. Conditions for which the other two roots become real (leading to a SN4) are determined by the discriminant of the polynomial. The respective expressions for different delay classes are included in Appendix C. The discriminant of $\sigma \equiv 0$ is omitted since, in this case, the control preserves the original SN4 $\left(\alpha=\alpha_{S N 4}\right)$. For the rest of $\sigma$ values, the SN4 could maintain after the cascade or approximate it coexisting with other periodic orbits.

Critical curves in the parameter plane $(\alpha, \eta)$ describing the regions where there exist solutions of period up to 4 for different $\sigma$ classes are depicted in Fig. 5. As expected, the first two branches of the period-doubling cascade as well as the P4 window inside chaos keep unchanged for any $\eta$ value and $\sigma \equiv 0$ [Fig. 5(a)]. Since this congruence class is composed of delay values equal to or greater than 4 , the period of the orbits in condition to be modified exceeds that of the solutions studied here.

Even delays belonging to class $\sigma \equiv 2(\bmod 4)$ permit to control the onset of P4 orbits [Fig. 5(b)]. For $\eta<0(\eta>0)$, the P4 branches arising in the period-doubling cascade shift to $\alpha \geq \alpha_{P 4}\left(\alpha \leq \alpha_{P 4}\right)$, increasing (decreasing) the interval where P2 orbits are stable. The SN4 curve moves to $\alpha$ values greater than $\alpha_{S N 4}$ for $\eta<0$ or $\eta>0.549$. However, it locates at $\alpha$ values lower than $\alpha_{S N 4}$ for $0<\eta \leq 0.549$. Inside this gain control interval, it could also occur that the map presents more than one SN4. The proximity of the curves plus the possibility of multiple SN4 solutions leads to the existence of bistable operations.

Figure 6 shows the dynamical scenario of the delayed map for $\eta=0.51$ and $\sigma=2$. For $\alpha \geq 3.4413$, stable P4 branches within the cascade coexist with a SN4. As the parameter is increased, the main stable P4 solution and the unstable one originated by the SN4 close together disappearing in a reverse SN4 bifurcation (at $\alpha=3.5133$ ). For $\alpha \geq 3.815$, a new SN4 adds to the unstable P4 solution generated by the previous interaction. As can be observed, analytical solutions (blue) are always in agreement with numerical simulations (red). Moreover, they permit to visualize the evolution of the unstable branches.

Odd delays not only control the interval where $\hat{y}_{-}$is stable, via the modification of the $\mathrm{P} 2$ curve, but also change the position of P4 orbits. As can be inferred from Figs. 5(c) and $5(d)$, the more intricate scenario is given by $\sigma \equiv 1(\bmod 4)$. 
(a)

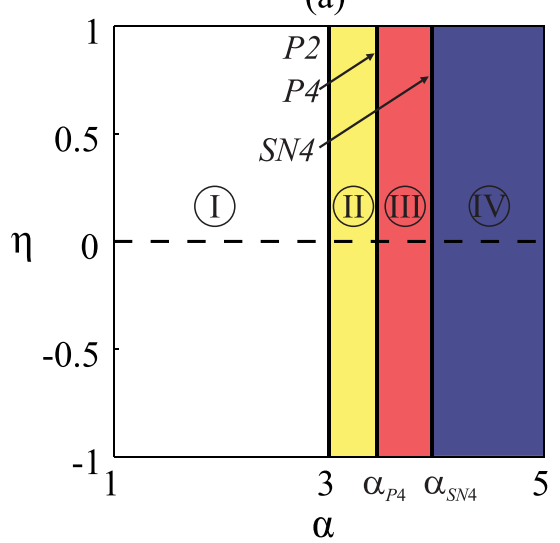

(c)

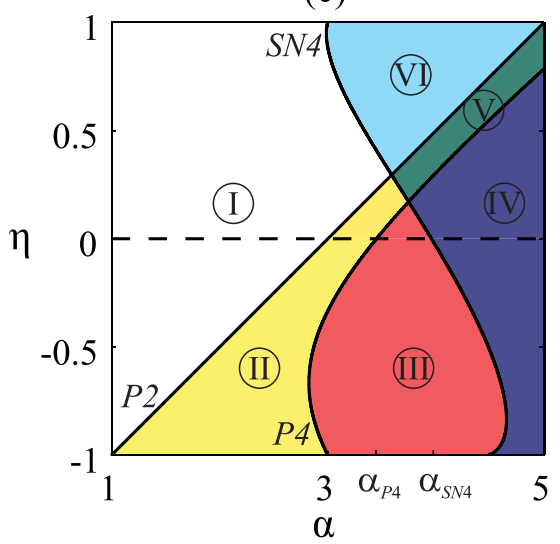

(b)

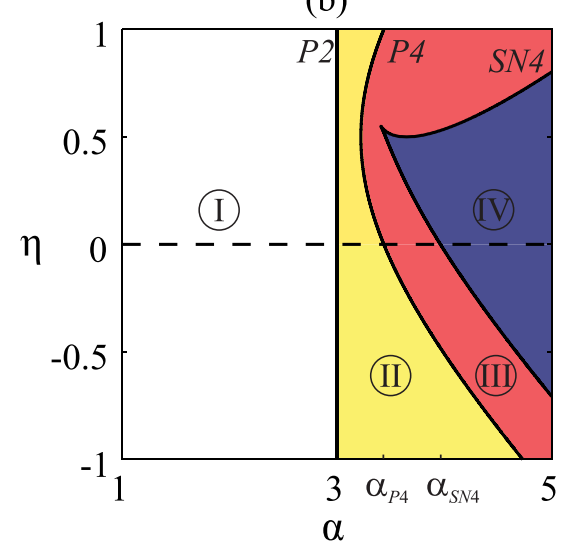

(d)

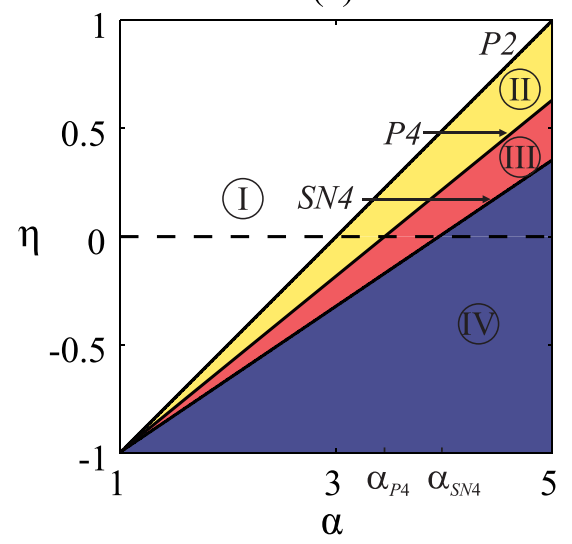

FIG. 5. Critical curves of P2 and P4 solutions for different delay values (mod4). (a) $\sigma \equiv 0$; (b) $\sigma \equiv 2$; (c) $\sigma \equiv 1$; (d) $\sigma \equiv 3$. I: fixed point (FP); II: FP + P2 solution; III: FP + P2 solution + one P4 solution; IV: FP + P2 solution + three P4 solutions; V: FP + P2 solution + two P4 solutions; VI: FP + two P4 solutions.
For $\eta<0, \mathrm{P} 2$ and $\mathrm{P} 4$ curves move to lower $\alpha$ values, but separating one from the other. Thus, the interval where $\mathrm{P} 2$ orbits are stable is increased while that where the fixed point is stable is reduced. The SN4 curve occurs for $\alpha \geq \alpha_{S N 4}$ maintaining the respective periodic window posterior to the perioddoubling cascade.

For $\eta>0, \mathrm{P} 2$ and $\mathrm{P} 4$ branches within the cascade shift similarly to greater $\alpha$ values. In contrast, SN4 curve moves to $\alpha \leq \alpha_{S N 4}$, approximating to the previous dynamics. In fact, SN4 passes through the P4 and even P2 critical curves as the gain is incremented. This analytical scenario confirms

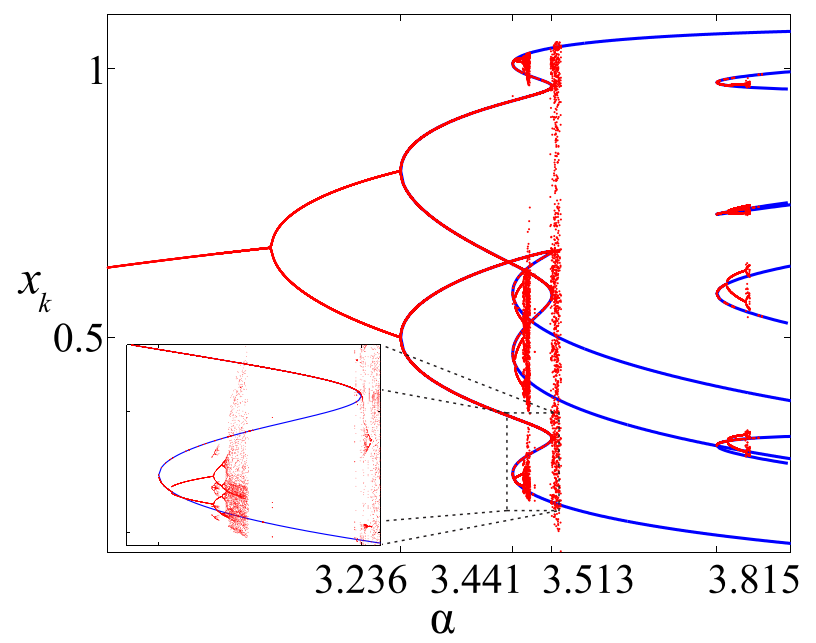

FIG. 6. Interaction of stable P4 solutions belonging to the period-doubling cascade with two SN4 bifurcations for $\sigma=2$ and $\eta=0.51$. Red: numerical simulations; blue: exact solutions obtained by the FD approach. that $\mathrm{P} 4$ attractors can coexist with the stable $\mathrm{P} 2$ orbits [region $\mathrm{V}$ in Fig. 5(c)] or the stable fixed point [region VI in Fig. $5(c)$, inducing again bistabilities in the controlled system. Figure 7 illustrates how the map can present a P2 orbit and two P4 orbits (one stable and one unstable) simultaneously for $\eta=0.2$ and $\sigma=1$.

The influence of the class $\sigma \equiv 3(\bmod 4)$ on the P2 and P4 orbits is almost linear [Fig. 5(d)]. For $\eta>0$, all critical curves shift to $\alpha$ values larger than those of the original map, extending the intervals where the fixed point and the P2 orbits are stable. The opposite occurs for $\eta<0$. The onset of

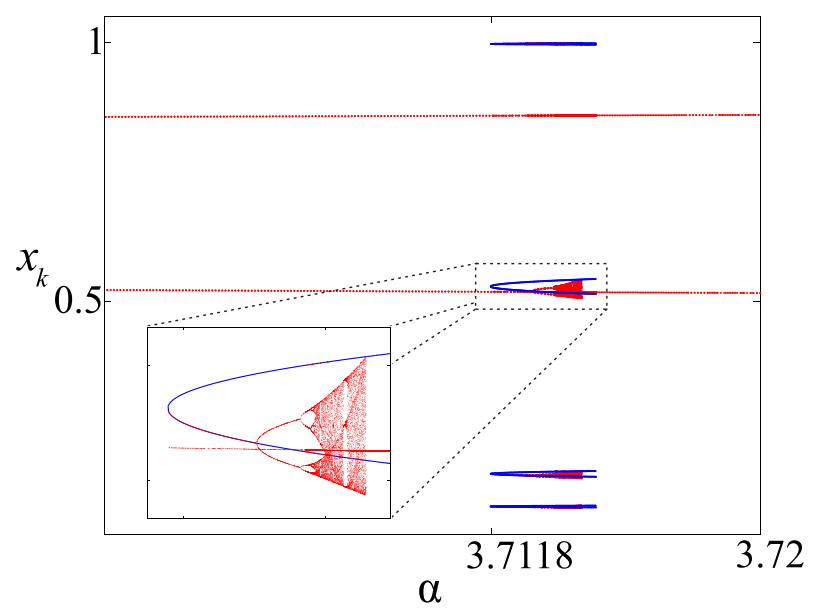

FIG. 7. Coexistence of stable P2 solutions with a SN4 bifurcation for $\sigma=1$ and $\eta=0.2$. Red: numerical simulations; blue: exact solutions obtained by the FD approach. 


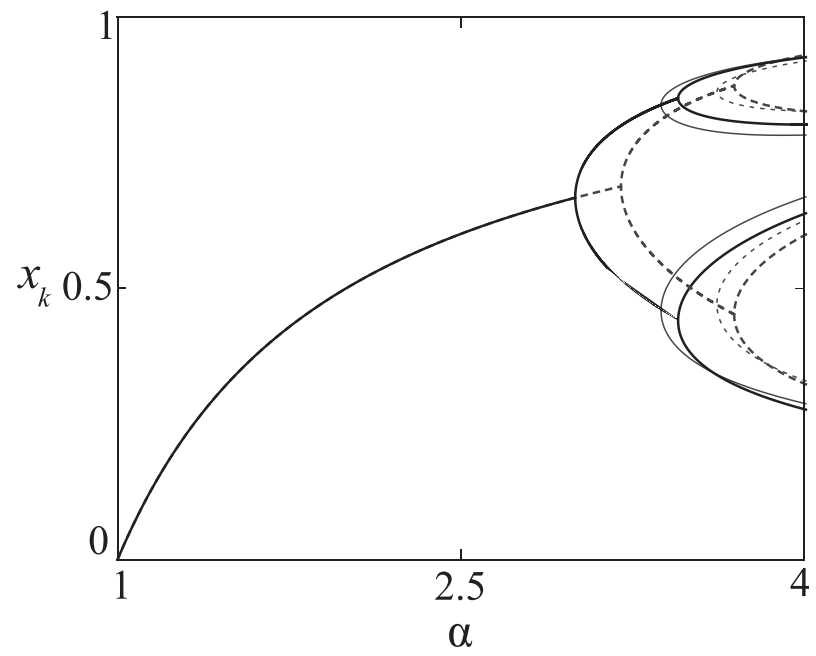

FIG. 8. Analytical solutions up to period-four of the delayed logistic map by considering $\eta=0.1$ and different delay values: (-) $\sigma \equiv 0$; (-) $\sigma \equiv 2$; (- -) $\sigma \equiv 1 ;(-) \sigma \equiv 3$

the solutions moves to lower $\alpha$ values. Moreover, since curves intersects at the point $(\alpha, \eta)=(1,-1)$, all the intervals are compressed as control gain is decreased.

Analytical solutions up to period-four obtained by considering the same control gain $(\eta=0.1)$ and different delay classes are depicted in Fig. 8. All curves given by $\sigma \equiv 0$ coincide with those of the uncontrolled logistic map (thick solid lines). For $\sigma \equiv 2$, the branch corresponding to the stable fixed point is kept equal to the original one $(\eta=0)$. However, stable P2 branches are reduced since the onset of the $\mathrm{P} 4$ orbits moves to smaller $\alpha$ values (thin solid lines). The interval where the fixed point is stable enlarges when odd delayed samples are used in the control law. Period-two branches do not present appreciable changes for $\sigma \equiv 1$ (thin dashed line). However, they occupy the largest parameter interval for $\sigma \equiv 3$ (thick dashed line), since the appearance of the P4 solutions is shift to greater $\alpha$ values. Delay class $\sigma \equiv 3$ seems to be appropriate to stabilize both fixed point and $\mathrm{P} 2$ orbits simultaneously.

\section{CONCLUSIONS}

Analytical and exact expressions of the period-four orbits exhibited by a family of $n$-dimensional quadratic maps have been obtained via a frequency-domain viewpoint. Critical conditions for the appearance of these periodic attractors have been also presented. Developments are based on a single-input single-output representation of the system, the complete Fourier series decomposition of the orbit, and the application of the harmonic balance method. The resulting set of four quadratic polynomial equations is solved thank to the use of Gröbner bases. A similar procedure could be follow by considering higher-periodic orbits. The greater the period, the more complex the resolution of the balance equations. The same could occur if maps are composed of higher-order nonlinearities.

A generalized scalar quadratic map and a delayed logistic model have been studied in detail to illustrate the applicability of the results. In the case of the controlled map, it has been shown that the stability range of the fixed point cannot be modified if an even number of delays is considered. Even delays congruent with two (modulus four) permit to shift the onset of period-four branches, increasing/decreasing the interval where period-two orbits are stable. As it has been described, this delay class can also cause that the map exhibits two stable period-four orbits simultaneously. All the oscillations with period up to four can be fully changed by using odd delays in the feedback law. However, it could occur that a period-four attractor coexists with the stable fixed point/period-two orbit if an odd delay value congruent with one (modulus four) is considered.

\section{ACKNOWLEDGMENTS}

The authors acknowledge the financial support of SGCyT at the Universidad Nacional del Sur (PGI 24/K064), Consejo Nacional de Investigaciones Científicas y Técnicas (PIP 112-201201-00062), and Agencia Nacional de Promoción Científica y Tecnológica (PICT 2014-2161).

\section{APPENDIX A: FD REPRESENTATION}

The application of the $z$-transform ${ }^{47}$ to the linear part of system (2) results in

$$
\begin{aligned}
z\left[X(z)-x_{0}\right] & =(A+B D C) X(z)+B E(z), \\
Y(z) & =C X(z),
\end{aligned}
$$

which is equivalent to

$$
\begin{gathered}
X(z)=[z I-(A+B D C)]^{-1} B E(z)+[z I-(A+B D C)]^{-1} x_{0} z, \\
Y(z)=C X(z),
\end{gathered}
$$

with $X(z), E(z)$, and $Y(z)$ standing for the $z$-transformation of $x_{k}, e_{k}$, and $y_{k}$, respectively, and $x_{0}$ representing the initial condition vector of $x_{k}$. Substituting (A1) into (A2), it is obtained

$$
\begin{aligned}
Y(z)= & C[z I-(A+B D C)]^{-1} B E(z) \\
& +C[z I-(A+B D C)]^{-1} x_{0} z .
\end{aligned}
$$

Then, defining $G(z)=C[z I-(A+B D C)]^{-1} B$ and considering that $x_{0}=0$, the output reduces to $Y(z)=G(z) E(z)$ as represented in Fig. 1.

\section{APPENDIX B: SINUSOIDAL RESPONSE OF THE LINEAR SUBSYSTEM}

Without loss of generality, let us suppose that the input of the linear subsystem is a sinusoid of the form $e_{k}=E_{1} \sin (\omega k)=E_{1}\left(e^{i \omega k}-e^{-i \omega k}\right) / 2 i$. Since its z-transform $^{47}$ is

$$
E(z)=\frac{E_{1} \sin (\omega) z}{\left(z-e^{i \omega}\right)\left(z-e^{-i \omega}\right)},
$$

output (A3) becomes 


$$
Y(z)=\frac{E_{1} \sin (\omega) z G(z)}{\left(z-e^{i \omega}\right)\left(z-e^{-i \omega}\right)}+C[z I-(A+B D C)]^{-1} x_{0} z .
$$

Taking into account that $G(z)$ and the term depending on $x_{0}$ have the same poles (which are different from $e^{i \omega}$ ), (B1) can be separated into two components, i.e., $Y(z)=Y_{e}(z)+Y_{g}(z)$, where $Y_{e}(z)$ and $Y_{g}(z)$ contain those terms of the general solution $Y(z)$ that originate in the poles of $E(z)$ and $G(z)$, respectively. ${ }^{48,49}$ If $G(z)$ is stable (all its poles have modulus less than one), the component of $y_{k}$ corresponding to the inverse $z$-transform of $Y_{g}(z)$ will tend naturally to zero with increasing $k$ and then the steady-state response is given by $Y_{e}(z)$. Nevertheless, it is always possible to find initial conditions so that $Y_{g}(z)$ can be forced to zero. Therefore, it can be assumed that the output $Y(z)$ generated in response to the sinusoid is directly $Y_{e}(z)$.

Based on (B1), this specific solution can be written as the partial fraction expansion ${ }^{47}$

$$
Y(z)=\frac{R z}{\left(z-e^{i \omega}\right)}+\frac{\bar{R} z}{\left(z-e^{-i \omega}\right)},
$$

where coefficient $R$ is given by

$$
R=\left.\frac{Y(z)\left(z-e^{i \omega}\right)}{z}\right|_{z=e^{i \omega}}=\left.\frac{E_{1} \sin (\omega) G(z)}{\left(z-e^{-i \omega}\right)}\right|_{z=e^{i \omega}}=\frac{E_{1} G\left(e^{i \omega}\right)}{2 i} .
$$

Then, applying the inverse $z$-transform to $Y(z)$, it results

$$
y_{k}=R e^{i \omega k}+\bar{R} e^{-i \omega k}=E_{1} \frac{G\left(e^{i \omega}\right) e^{i \omega k}-G\left(e^{-i \omega}\right) e^{-i \omega k}}{2 i} .
$$

Notice that the response of $G\left(e^{i \omega}\right)$ is conjugate-symmetric with respect to zero, i.e., its value at $-\omega$ is the complex conjugate of that at $+\omega$. This is due to the fact that replacing $\omega$ by $-\omega$ gives the same result as replacing $i$ by $-i$ in $e^{i \omega}$. For that reason, output $y_{k}$ can be reduced to the sinusoid

$$
y_{k}=\left|Y_{1}\right| \sin \left(\omega k+\angle Y_{1}\right)=\left|Y_{1}\right| \frac{e^{i\left(\omega k+\angle Y_{1}\right)}-e^{-i\left(\omega k+\angle Y_{1}\right)}}{2 i},
$$

with coefficient $Y_{1}$ defined as $Y_{1}=G\left(e^{i \omega}\right) E_{1}$. As can be seen, if the input of the linear block is a sinusoid of frequency $\omega$, the output response will be a sinusoid of the same frequency. ${ }^{48,49}$ The amplitude of the new sinusoid will be $\left|G\left(e^{i \omega}\right)\right|$ times the amplitude of the original one, and its phase will be equal to the phase of the input plus $\angle G\left(e^{i \omega}\right)$.

A similar procedure can be followed by considering an input $e_{k}$ composed of a sum of sinusoidal terms. Based on the superposition principle for linear systems, it can be found that the particular solution $y_{k}$ will be a sum of the same terms but modified in amplitude and angle by $G(z)$ evaluated at the respective frequencies. This property suggests also that if $y_{k}=\sum_{r=0}^{p}\left|Y_{r}\right| \sin \left(r \omega k+\angle Y_{r}\right)$ and the corresponding input is $e_{k}=\sum_{r=0}^{p}\left|E_{r}\right| \sin \left(r \omega k+\angle E_{r}\right)$, then $Y_{r}=G\left(e^{i r \omega}\right) E_{r}$ with $r=0, \ldots, p$. Hence, it results possible to define the so-called harmonic balance equations (9)-(10) and (17)-(19) presented in Section III.

\section{APPENDIX C: EXPRESSIONS CORRESPONDING TO THE DISCRIMINANT OF THE CUBIC POLYNOMIAL IN $\tilde{Y}_{0}$ FOR DIFFERENT $\sigma$ CLASSES $(\bmod 4)$.}

$$
\begin{aligned}
& \text { For } \sigma \equiv 1, \\
& \begin{aligned}
0= & -135-54 \alpha-9 \alpha^{2}+28 \alpha^{3}+3 \alpha^{4}-6 \alpha^{5}+\alpha^{6} \\
& +12\left(27+12 \alpha-2 \alpha^{2}-4 \alpha^{3}+\alpha^{4}\right) \eta-12(12+20 \alpha \\
& \left.-6 \alpha^{2}-4 \alpha^{3}+\alpha^{4}\right) \eta^{2}-16\left(5-12 \alpha+6 \alpha^{2}\right) \eta^{3} \\
& -12\left(19-8 \alpha-4 \alpha^{2}\right) \eta^{4}+408 \eta^{5}-172 \eta^{6} .
\end{aligned}
\end{aligned}
$$

For $\sigma \equiv 2$,

$$
\begin{aligned}
0= & -135-54 \alpha-9 \alpha^{2}+28 \alpha^{3}+3 \alpha^{4}-6 \alpha^{5}+\alpha^{6} \\
& -24\left(36+12 \alpha-2 \alpha^{2}-4 \alpha^{3}+\alpha^{4}\right) \eta-12(192+44 \alpha \\
& \left.-18 \alpha^{2}-4 \alpha^{3}+\alpha^{4}\right) \eta^{2} \\
& -16\left(203+24 \alpha+12 \alpha^{2}\right) \eta^{3}-4\left(633+24 \alpha-12 \alpha^{2}\right) \eta^{4} \\
& -1032 \eta^{5}-172 \eta^{6} .
\end{aligned}
$$

For $\sigma \equiv 3$,

$$
\begin{aligned}
0= & -135-54 \alpha-9 \alpha^{2}+28 \alpha^{3}+3 \alpha^{4}-6 \alpha^{5}+\alpha^{6} \\
& +12\left(9-24 \alpha+8 \alpha^{2}+4 \alpha^{3}-\alpha^{4}\right) \eta-8\left(63-12 \alpha+10 \alpha^{2}\right. \\
& \left.-4 \alpha^{3}+\alpha^{4}\right) \eta^{2}+32\left(9-4 \alpha+2 \alpha^{2}\right) \eta^{3} \\
& -16\left(7+2 \alpha-\alpha^{2}\right) \eta^{4}-64 \eta^{5} .
\end{aligned}
$$

${ }^{1}$ R. May, "Simple mathematical models with very complicated dynamics," Nature 261, 459-475 (1976).

${ }^{2}$ R. L. Devaney, A First Course in Chaotic Dynamical Systems (AddisonWesley Publishing Inc., MA, 1992).

${ }^{3}$ R. V. Solé and J. Valls, "On structural stability and chaos in biological systems," J. Theor. Biol. 155, 87-102 (1992).

${ }^{4}$ M. E. Brandt and G. Chen, "Feedback control of a quadratic map model of cardiac chaos," Int. J. Bifurcation Chaos 6, 715-723 (1996).

${ }^{5}$ K. Hall, J. D. Christini, M. Tremblay, J. J. Collins, L. Glass, and J. Billette, "Dynamic control of cardiac alternans," Phys. Rev. Lett. 78, 4518-4521 (1997).

${ }^{6}$ T. Quail, N. McVicar, M. Aguilar, M.-Y. Kim, A. Hodge, L. Glass, and A. Shrier, "Chaotic dynamics in a cardiac aggregates induced by potassium channel block," Chaos 22, 033140 (2012).

${ }^{7}$ H. N. Agiza and A. A. Elsadany, "Chaotic dynamics in nonlinear duopoly game with heterogeneous players," Appl. Math. Comput. 149, 843-860 (2004).

${ }^{8}$ L. Zachilas and A. Gkana, "Nonlinear supply and demand model with two interrelated markets," J. Eng. Sci. Technol. Rev. 4, 227-232 (2011).

${ }^{9} \mathrm{G}$. W. Luo and J. H. Xie, "Bifurcations and chaos in a system with impacts," Physica D 148, 183-200 (2001).

${ }^{10} \mathrm{G}$. W. Luo and J. H. Xie, "Stability of periodic motion, bifurcations and chaos of a two-degree-of-freedom vibratory system with symmetrical rigid stops," J. Sound Vib. 273, 543-568 (2004).

${ }^{11}$ D. Dangoisse, P. Glorieux, and D. Hennequin, "Chaos in a $\mathrm{CO}_{2}$ laser with modulated parameters: Experiments and numerical simulations," Phys. Rev. A 36, 4775-4791 (1987).

${ }^{12}$ D. Pieroux and T. Erneux, "Minimal model of a class-B laser with delayed feedback: Cascading branching of periodic solutions and period-doubling bifurcation," Phys. Rev. A 50, 1822-1829 (1994).

${ }^{13}$ B. E. Martínez-Zérega, A. N. Pisarchik, and L. S. Tsimring, "Using periodic modulation to control coexisting attractors induced by delayed feedback," Phys. Lett. A 318, 102-111 (2003).

${ }^{14}$ C. K. Tse, Complex Behavior of Switching Power Converters (CRC Press, Boca Raton, 2004). 
${ }^{15}$ A. E. Aroudi, M. Debbat, R. Giral, G. Olivar, L. Benadero, and E. Toribio, "Bifurcations in dc-dc switching converters: Review of methods and applications," Int. J. Bifurcation Chaos 15, 1549-1578 (2005).

${ }^{16}$ E. Sander and J. A. Yorke, "Connecting period-doubling cascades to chaos," Int. J. Bifurcation Chaos 22, 1250022 (2012).

${ }^{17}$ E. Sander and J. A. Yorke, "A period-doubling cascade precedes chaos for planar maps," Chaos 23, 033113 (2013).

${ }^{18}$ Y. A. Kuznetsov, Elements of Applied Bifurcation Theory, 3rd ed. (Springer-Verlag, New York, 2004).

${ }^{19} \mathrm{M}$. Peng, "Multiple bifurcations and periodic bubbling in a delay population model," Chaos, Solitons Fractals 25, 1123-1130 (2005).

${ }^{20}$ J. C. Sprott, Chaos and Time-Series Analysis (Oxford University Press, New York, 2001).

${ }^{21}$ G. Pastor, M. Romera, A. B. Orue, A. Martin, M. F. Danca, and F. Montoya, "Harmonic analysis in discrete dynamical systems," Int. J. Mod. Nonlinear Theory Appl. 1, 14-31 (2012).

${ }^{22}$ D. L. Hitzl and F. Zele, "An exploration of the Hénon quadratic map," Physica D 14, 305-326 (1985).

${ }^{23}$ A. Endler and J. A. C. Gallas, "Period four stability and multistability domains for the Hénon map," Physica A 295, 285-290 (2001).

${ }^{24}$ A. Endler and J. A. C. Gallas, "Arithmetical signatures of the dynamics of the Hénon map,” Phys. Rev. E 65, 036231 (2002).

${ }^{25}$ P. E. Phillipson, "Analytics of period doubling," Commun. Math. Phys. 111, 137-149 (1987).

${ }^{26}$ M. D'Amico, J. Moiola, and E. Paolini, "Hopf bifurcation for maps: A frequency domain approach," IEEE Trans. Circuits Syst. 49, 281-288 (2002).

${ }^{27}$ M. D'Amico, J. Moiola, and E. Paolini, “A frequency domain method for analyzing period doubling bifurcations in discrete-time systems," Circuits Syst. Signal Process. 23, 517-536 (2004).

${ }^{28}$ M. B. D'Amico, J. L. Moiola, and E. E. Paolini, "Study of degenerate bifurcations in maps: A feedback system approach," Int. J. Bifurcation Chaos 14, 1625-1641 (2004).

${ }^{29}$ D. J. Allwright, "Harmonic balance and the Hopf bifurcation theorem," Math. Proc. Cambridge Philos. Soc. 82, 453-467 (1977).

${ }^{30}$ A. I. Mees and L. O. Chua, "The Hopf bifurcation theorem and its applications to nonlinear oscillations in circuits and systems," IEEE Trans. Circuits Syst. 26, 235-254 (1979).

${ }^{31}$ A. Mees, Dynamics of Feedback Systems (John Wiley \& Sons, Chichester, 1981).

${ }^{32}$ J. Moiola and G. Chen, Hopf Bifurcation Analysis: A Frequency Domain Approach (World Scientific Publishing Co., Singapore, 1996).
${ }^{33}$ A. Tesi, E. Abed, R. Genesio, and H. Wang, "Harmonic balance analysis of period-doubling bifurcations with implications for control of nonlinear dynamics," Automatica 32, 1255-1271 (1996).

${ }^{34} \mathrm{G}$. R. Itovich and J. L. Moiola, "Double Hopf bifurcation analysis using frequency domain methods," Nonlinear Dynam. 39, 235-258 (2005).

${ }^{35}$ F. S. Gentile, J. L. Moiola, and E. E. Paolini, "On the study of bifurcations in delay-differential equations: A frequency-domain approach," Int. J. Bifurcation Chaos 22, 1250137 (2012).

${ }^{36}$ A. Torresi, G. Calandrini, P. Bonfili, and J. Moiola, "Generalized Hopf bifurcation in a frequency domain formulation," Int. J. Bifurcation Chaos 22, 1250197 (2012).

${ }^{37}$ D. Cox, J. Little, and D. O'Shea, Ideals, Varieties, and Algorithms, 3rd ed. (Springer, New York, 2007).

${ }^{38}$ T. Hisakado and K. Okumura, "Mode decomposition of global bifurcation diagram with Gröbner bases,” Phys. Lett. A 292, 263-268 (2002).

${ }^{39}$ I. S. Kotsireas and K. Karamanos, "Exact computation of the bifurcation point $B_{4}$ of the logistic map and the bailey-broadhurst conjectures," Int. J. Bifurcation Chaos 14, 2417-2423 (2004).

${ }^{40}$ Period-two solutions are included for completeness since they are used in Sec. III B.

${ }^{41}$ Let the ring of polynomials $R=K\left[x_{1}, \ldots, x_{n}\right]$, a subset $I \subset K\left[x_{1}, \ldots, x_{n}\right]$ is an ideal if it satisfies: (i) $0 \in I$; (ii) if $f, g \in I$ then $f+g \in I$; (iii) if $f \in I$ and $h \in k\left[x_{1}, \ldots, x_{n}\right]$, then $h+f \in I$.

${ }^{42}$ F. Gentile, A. Bel, M. D'Amico, and J. Moiola, "Effect of delayed feedback on the dynamics of a scalar map via a frequency-domain approach," Chaos 21, 023117 (2011).

${ }^{43}$ I. M. Gelfand, M. M. Kapranov, and A. V. Zelevinsky, Discriminants, Resultants and Multidimensional Determinants (Birkhäuser, Boston, 1994).

${ }^{44}$ K. Pyragas, "Continuous control of chaos by self-controlling feedback," Phys. Lett. A 170, 421-428 (1992).

${ }^{45}$ To be precise, the delay in time is chosen according to the period of the orbit to be stabilized.

${ }^{46}$ T. Buchner and J. J. Żebrowski, "Logistic map with a delayed feedback: Stability of a discrete time-delay control of chaos," Phys. Rev. E 63, 016210 (2000).

${ }^{47}$ G. James, Advanced Modern Engineering Mathematics, 4th ed. (Prentice Hall, Harlow, 2011).

${ }^{48}$ C. L. Phillips and H. T. Nagle, Digital Control Systems, Analysis and Design, 3rd ed. (Prentice Hall, NJ, 1995).

${ }^{49}$ R. J. Vaccaro, Digital Control: A State-Space Approach (McGraw-Hill, New York, 1995). 